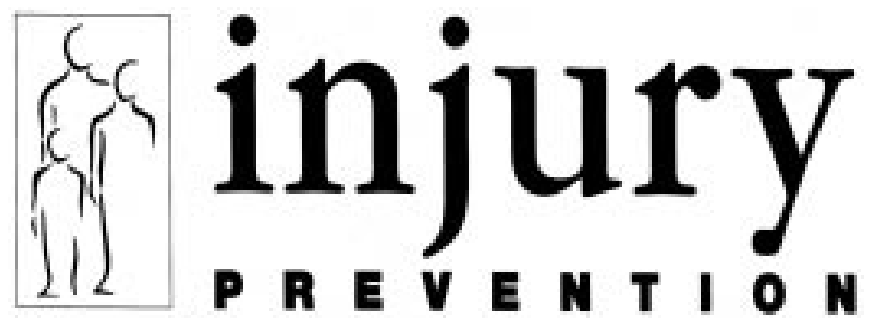

\title{
Editorial
}

\section{Successes and failures in occupational injury prevention}

Occupational accidents are a major health hazard. According to International Labor Office, injuries killed 335000 people at work in 1994. ${ }^{1}$ The good news is that there is a declining trend of fatal accidents in most industrialized countries. For too many, injuries are, however, like a fringe "benefit". If one gets a job, one also has to accept the risk of being killed or seriously injured at work.

Another element of good news is that the best companies have reduced their injury rates to very low levels. Especially the petrochemical industry, which has improved its safety performance in an outstanding manner. Many other industries are also putting serious efforts into promoting safety. For example, the steel industry recently published a book guiding steel companies towards "accident free production". 2

Ethical issues are also receiving more attention in the global business world. Several events during the past two years have shown that many people mistrust global corporations. The business world counteracts positively by emphasizing ethical principles. Occupational injuries or other ill health arising from work are not deemed acceptable if a company claims to be following high ethical standards.

Many people may not believe in voluntary ethical standards. In safety, we too often have to accept the good results of voluntary efforts. And, to be fair, at least some companies have excellent safety practices. For example, the chemical industry has been using the global "Responsible Care" program and this has lead to many examples of excellent safety at work. To better promote safety in the workplace, we need to better identify exemplary, landmark companies.

The development of better communication systems appears to have had a positive effect on safety in the workplace. Benchmarking has also become popular. This permits companies to continually compare their performance with that of other companies, not only in the same country but also in other countries.

Comparisons between countries are thus a critically important tool to support the creation of nationwide preventive programs. Therefore the paper by Feyer and her colleagues in this issue is an important step towards better understanding the effectiveness of prevention. ${ }^{3}$ In this paper, work related fatal injuries in the United States, Australia, and New Zealand are compared. The rate of fatal occupational injuries was highest in New Zealand and lowest in the United States. Much of this difference is accounted for by the differences in the structure of industry. For example, water transport injuries are more prominent in New Zealand, and this is almost certainly a matter of exposure.

One surprising finding was that the largest difference among these countries was in the rate of intentional injuries in the workplace. Employees in the United States are subject to intentional injury much more often than in the other two countries. Homicide is most frequent in sales jobs. However, managers, professionals, and people working for public administration also experience violent injuries more often in the United States than in the other two countries.

Differences are not the only interesting aspect in these international comparisons. Similarities are at least as important. It is noteworthy that mining, agriculture, forestry, and construction are in all three countries the work settings where there is the highest probability of being killed in a work related injury. Machinery, falling objects, and falls are consistently the leading causes of these fatalities.

Why is it that the construction industry has high injury rates everywhere, while the manufacturing industry has been able to reduce rates considerably? The variability of working conditions is likely to be one answer. Yet, the manufacturing industry produces a wide variety of products. For example, the auto industry assembles different models with different colors and equipment packages on the same production lines.

Another difference between the manufacturing industry and the construction industry is specialization. The manufacturing sector is highly specialized according to products and specialization continues, for example, in the form of outsourcing. The construction industry is much less specialized and has not developed standardized production processes.

A further difference between manufacturing and construction is that manufacturing firms are much more international and, therefore, more transparent than construction firms. Construction has not faced similar competition as manufacturing. Agriculture is another sector of activity displaying high injury rates internationally and agriculture is also much more domestic than manufacturing in all countries.

Feyer and her colleagues restricted their study to fatal injuries. Yet, they faced many problems in collecting consistent datasets from all three countries. These difficulties would be even greater if less serious injuries had been 
included. In spite of difficulties, we must continue to conduct international comparisons to learn more about success and failure in prevention. Why, for example, has the petrochemical industry in some countries been able to lower injury rates much more than in others?

"Safety culture" became a popular concept among safety researchers during the last decade. ${ }^{4}$ The three countries Feyer et al studied were culturally different but similar in many other respects. However, their results quite clearly seem to reflect structural differences more than cultural differences. Is it that with safety culture we have invented "a face saving concept"? If we do not understand (or accept) what needs to be done to prevent injuries, one can always withdraw behind the mysterious "safety culture". As culture changes so slowly, we cannot be blamed for not doing anything even if we actually do not know what to do... or so goes the rationalization.

This rather cynical view of safety culture may not be justified. Perhaps there is a widespread culture about safety that includes a large dose of fatalism. As the same picture of work related fatal injuries prevails everywhere, there should be something in common. Common structural factors may the connecting factor. For example, agriculture is a family business in most countries.

In addition to structural factors, there may also be common cultural factors. One could be the lack of belief in prevention. This may be universal and extend to other health areas, too. Finding success stories is essential to convince people about the feasibility and necessity of prevention.

In the area of injury, we need to convince others that prevention is both important and feasible. Another thought provoking paper in this issue strongly supports this simple statement. O'Connor's article is about work related spinal cord injury. ${ }^{5}$ Such injuries are fortunately rare; there is no cure and the level of impairment does not improve substantially even after rehabilitation. Therefore, prevention is the only way to go.

Falls are a common cause of spinal cord injuries and, of course, of many other types of injuries as well. As many people seem to be quite fatalistic about the possibility of preventing falls, we need more research on walking safely and maintaining body balance.

Spinal cord injuries have a substantial economic effect. According to O'Connor, spinal cord injuries generated a long term care cost of more than 23 million Australian dollars each year. The long term care cost for a ventilator dependent tetraplegic is $\$ 4$ million (Australian). It would seem that this shocking figure alone readily justifies even an expensive preventive program.

In my opinion, the cost of injuries should not be the driving force of prevention: rather, it should be the improvement of the quality of life. Yet, these examples also demonstrate the economic value of injury prevention. Money saved is a bonus from successful injury prevention.

Finnish Institute of Occupational Health,

JORMA SAARI

Laajaniityntie 1, Fin-01620 Vantaa,

Finland

1 Takala J. Global estimates of fatal occupational accidents. ILO sixteenth international conference of labour statisticians, 6-15 October 1998. Geneva: International Labour Office, 1998.

2 International Iron and Steel Institute. Accident-free steel. London: International Iron and Steel Institute, 1998.

3 Feyer A-M, Williamson AM, Stout N, et al. Comparison of work related fatal injuries in the United States, Australia, and New Zealand: method and overall findings. Inj Prev 2001;7:22-8.

4 Hale AR, Hovden J. Management and culture: the third age of safety. A review of approaches to organizational aspects of safety, health and environment. In: Feyer AM, Williamson A, eds. Occupational injury: risk, prevention and intervention. London: Taylor \& Francis, 1998: 129-67.

5 O'Connor P. Work related spinal cord injury, Australia 1986-97. Inj Prev 2001;7:29-34.

\section{Guest editorial}

\section{Research priorities for injury prevention}

The aim of injury prevention research is to provide information that will lead to a sustained reduction in the burden of injury, whether measured in terms of mortality, morbidity, disability, or cost of injury. Because the resources available for injury research are limited, funding for injury prevention research must be rationed. A question therefore arises about the criteria that should be used to decide how the available research funding should be allocated to the different types of research activities. If we agree with the aim stated above, it would seem reasonable to prioritise research that provides the greatest marginal benefit, in terms of reduction in the burden of injury, for any given cost. In other words, priority setting should be driven by a comparison of incremental gains with incremental costs. Because resources for injury research are limited we should fund the most cost effective injury prevention research.

The outcome of research is the provision of information. But how can we predict what type of information will lead to the greatest reduction in the burden of injury? We can approach this question by considering how information from injury prevention research would lead to a reduction in the burden of injury. Injury research will only contribute to the reduction in the burden of injury if it informs the actions we take for injury prevention. The information we need is that which would steer us towards effective and cost effective action and away from action that is harmful, useless, or of low cost effectiveness. Research on the effectiveness and cost effectiveness of injury prevention interventions might therefore be expected to represent a good buy for research funders. This is not to say that other research activities are a waste of money. Clearly, a range of research methodologies can influence actions taken for injury prevention. Case-control and cohort studies, by identifying modifiable risk factors for injury, provide a focus for injury prevention activities, and qualitative research can inform the design of prevention programmes. However, casecontrol and cohort studies at best identify associations with injury occurrence and tell us nothing about the effectiveness of intervention. The knowledge that alcohol is a strong risk factor for motor vehicle crashes tells us nothing about the effectiveness of random breath testing in reducing road 
deaths and injuries. The final stage in the information chain leading to injury reduction will always be the evaluation of the effectiveness of injury prevention interventions. Because information on effectiveness is closer to injury reduction than information on risk factors, and because we value current benefits more highly than future benefits, the provision of information on effectiveness and cost effectiveness should be a priority for injury prevention research.

A second criterion for prioritising research is that the information provided should be valid. Biased studies provide misleading information on the effectiveness and cost effectiveness of interventions and may therefore expose policy makers to the risk of wasting resources on useless interventions. Because the validity of information from randomised controlled trials is more readily assured than with other study designs, they represent good value for money for injury research funders. Randomised controlled trials of the effectiveness of injury prevention interventions provide the most highly valued information and the most valid information.

If we accept that information from randomised controlled trials of the effectiveness of injury prevention interventions represents the best buy for research funders how can this information be provided for least possible cost. One strategy is to make the best use of the currently available effectiveness information by identification and synthesis of the results of controlled evaluation studies in the past. Interventions that have been shown to be effective in one setting are likely to be effective elsewhere and it may be more efficient to find out what the results of evaluation studies conducted in other parts of the world show before embarking on new studies. For a problem as common as injury, even moderate intervention effects can be important. However, moderate effects are difficult to detect reliably without large amounts of evidence. This evidence may best be obtained by a systematic review and meta-analysis of the results from controlled evaluation studies in the past.

A second strategy for reducing the cost of providing effectiveness information is to orchestrate the implementation of existing injury prevention interventions in a way that facilitates rigorous evaluation. For example, as part of a community based initiative to prevent fire deaths, the Camden \& Islington Accident Prevention Alliances proposed to distribute free smoke alarms to disadvantaged families in high risk inner city housing. There was not enough money to provide free alarms for all residents and the effectiveness of giveaway programmes, although promising, had yet to be established. Alarms were therefore distributed in the context of a randomised controlled trial, half the wards received free alarms, the other half served as a comparison group. This approach facilitated the provision of rigorous information on the cost effectiveness of giving away smoke alarms while at the same time providing an equitable way of rationing a scarce resource. Because the resources available for injury prevention activities are limited in the same way as resources are limited for injury research, there are always opportunities for conducting randomised controlled trials in response to the dual demands of resource rationing and rigorous evaluation.

So far there has been no reference to the value of injury surveillance in the provision of information for injury control. Injury surveillance entails counting the numbers and characteristics of injured persons, sometimes taking account of the severity and cost of the injuries sustained. Injury surveillance is considered to be of value in establishing injury prevention priorities, targeting injury prevention activities, and monitoring progress towards injury reduction goals. In establishing priorities and targeting, surveillance data may be of some value but it makes more sense to prioritise and target injury prevention activities on the basis of the marginal benefit compared with marginal cost. For example, compared with road traffic crashes, residential fires account for a relatively small number of injury deaths. Nevertheless, if there was an effective strategy for preventing fire deaths such that the cost per life saved was much less than for any road safety intervention, preventing fire deaths would represent a more rational use of existing resources. In monitoring progress towards injury reduction goals, the use of injury surveillance is flawed for obvious scientific reasons.

Whether injury rates rise or fall implies nothing about the value of our prevention activities. The determinants of the injury rate include economic and social factors over which practitioners and even governments have limited control. A rise in injury occurrence is quite consistent with the implementation of an effective injury prevention intervention.

There are, however, two important roles for injury surveillance. The first is in the evaluation of injury prevention interventions where injury surveillance can facilitate the collection of outcome data in controlled evaluation studies. In this case, the surveillance system should be tailored towards to the needs of the trial in question and should be discontinued once the evaluation study is completed. The second is in monitoring the implementation of effective prevention programmes. If smoke alarm installation was shown to be effective in reducing fire deaths or injuries in randomised controlled trials, it would be more useful to monitor the proportion of homes with alarms installed than to monitor fire deaths or injuries. In this situation, information on alarm installation would be of immediate relevance to policy makers as it would indicate where efforts should be directed. Information on fire deaths and injuries on the other hand would tell policy makers very little.

Department of Epidemiology and Biostatistics,

IAN ROBERTS

Institute of Child Health,

University of London,

30 Guilford Street,

London WC1N 1EH, UK

ian.roberts@ich.ucl.ac.uk 